\title{
Desnutrición crónica y anemia en niños menores de 5 años de hogares indígenas del Perú - Análisis de la Encuesta Demográfica y de Salud Familiar 2013
}

Chronic malnutrition and anemia in children under 5 years of indigenous households of Peru - Analysis of Demographic and Health Survey 2013

\author{
Janet Flores-Bendezú ${ }^{1}$, Juan Calderón ${ }^{1}$, Betty Rojas ${ }^{1}$, Edith Alarcón-Matutti ${ }^{2}$,
} César Gutiérrez

'Programa de Segunda Especialidad en Nutrición Pública, Facultad de Medicina, Universidad Nacional Mayor de San Marcos, Lima, Perú.

${ }^{2}$ Departamento Académico de Medicina Preventiva y Salud Pública, Facultad de Medicina, Universidad Nacional Mayor de San Marcos,

Lima, Perú.

\begin{abstract}
Resumen
Introducción: La desnutrición crónica y anemia infantil son un problema importante de salud pública, cuyas consecuencias se manifiestan a lo largo de todo el ciclo vital. El Estado peruano se caracteriza por albergar una de las mayores riquezas etnoculturales del continente americano, siendo la Amazonía Peruana la región que posee la mayor diversidad de grupos indígenas del país. Objetivo: Determinar la prevalencia de desnutrición crónica y anemia en niños menores de 5 años de hogares indígenas del Perú. Diseño: análisis secundario de los datos de la Encuesta Demográfica y de Salud Familiar (ENDES) 2013, del Instituto Nacional de Estadística e Informática (INEI). Institución: Segunda Especialidad en Nutrición Pública, Facultad de Medicina, Universidad Nacional Mayor de San Marco. Lima, Perú. Participantes: Niños menores de 5 años de hogares de la región natural selva en los cuales el idioma principal es una lengua indígena. Principales medidas de resultados: Desnutrición crónica (<-2 DE para valores Z de talla para la edad) y anemia ( $<11 \mathrm{~g} / \mathrm{dL}$ de hemoglobina ajustado por altitud). Resultados: La desnutrición crónica afectó al $43,0 \%$ y la anemia al $43,5 \%$ de los niños menores de 5 años de hogares indígenas. No existió una asociación estadísticamente significativa entre desnutrición crónica con el sexo ni edad del niño, ni entre la anemia y sexo del niño. Sin embargo, sí se encontró asociación entre la edad del menor y la anemia $(p<0,001)$. Conclusiones: La desnutrición crónica y anemia resultaron elevadas en niños menores de 5 años de hogares indígenas en la selva del Perú, siendo evidentes las grandes desigualdades en la situación de pobreza, servicios básicos y salud de los niños indígenas.
\end{abstract}

Palabras clave: Desnutrición, anemia, población indígena.

\section{Abstract}

Introduction: Child chronic malnutrition and anemia represent a major public health problem whose consequences are manifested throughout the life cycle. The Peruvian State is characterized by hosting one of the largest ethno-cultural wealth of the Americas, being the Peruvian Amazon region the one that has the greatest diversity of indigenous groups in the country. Objective: To determine the prevalence of chronic malnutrition and anemia in children under 5 years of indigenous households in Peru. Design: Secondary analysis of data from the Demographic and Health Survey (DHS) 2013, National Institute of Statistics and Informatics. Institution: Second Specialization in Public Nutrition, Faculty of Medicine, Universidad Nacional Mayor de San Marcos, Lima, Peru. Participants: Children under 5 years of households from the Amazon region where the primary language is indigenous. Main outcome measures: Chronic malnutrition ( $<-2$ Z-scores for height for age) and anemia ( $<11 \mathrm{~g} / \mathrm{dL}$ hemoglobin adjusted for altitude). Results: Chronic malnutrition affected $43 \%$ and anemia $43.5 \%$ of children under 5 years of indigenous households. There was no statistically significant association between chronic malnutrition and sex or age of the child, nor between anemia and gender of the child; however an association between the child's age and anemia was found $(p<0.001)$. Conclusions: Chronic malnutrition and anemia were high in children under 5 years of indigenous households in the Amazon region of Peru, evidencing the large disparities in poverty, basic services and health in indigenous children.

Keywords: Malnutrition, anemia, indigenous population.

An Fac med. 2015;76(2):135-40 / dx.doi.org/10.15381/anales.v76i2.11139

\section{INTRODUCCIÓN}

La desnutrición crónica y anemia infantil son un importante problema de salud pública, cuyas consecuencias se manifiestan a lo largo de todo el ciclo vital y que afecta principalmente a los pobres o pobres extremos. De ahí la importancia de enfrentar la desnutrición crónica porque tiene efectos negativos inmediatos, como mayor probabilidad de ocurrencia de enfermedades o muerte prematura en niños menores de cinco años, y a largo plazo afecta el desempeño escolar, la capacidad de trabajo y ocasiona costos y pérdidas económicas a la familia y la sociedad ${ }^{(1-3)}$.
El Estado peruano se caracteriza por albergar una de las mayores riquezas etnoculturales del continente americano y del mundo. El Instituto Nacional de Estadística e Informática (INEI, 1993 y 2009) señala que la Amazonía es la región que posee la mayor diversidad de grupos indígenas del país, culturalmen- 
te diferenciadas con su propia lengua, música, arquitectura, manifestaciones etnográficas, tradiciones milenarias, mitología y conocimientos médicos naturales, entre otros. Sin embargo, los pueblos indígenas amazónicos empezaron a sufrir, y aún siguen sufriendo, el impacto de la colonización, de la construcción de carreteras, de los grandes y pequeños extractores de recursos naturales y de los culturizadores que piensan que para ser reconocidos como ciudadanos deben adoptar los modelos de la cultura occidental ${ }^{(4-5)}$. Los resultados de los Censos Nacionales 2007, XI de Población y VI de Vivienda indican que la población indígena de la Amazonía fue de 332975 habitantes y está compuesta por 51 pueblos étnicos divididos en aproximadamente 13 familias lingüísticas. Estas son los Arahuaca, $\mathrm{Ca}$ huapana, Harakmbut, Huitoto, Jíbaro, Pano, Peba Yagua, Quechua, sin clasificación, Tacana, Tucano, Tupi-Guaraní y los Záparo. Dentro de estas familias lingüísticas, las etnias con mayor población fueron los Asháninka (23,0\%), Aguaruna (15,7\%), Quechua (7,3\%) y Chayahuita $(6,9 \%)$. Como dato resaltante se observa que aproximadamente la quinta parte de la población $(17,9 \%)$ es menor a 5 años, determinando así una pirámide poblacional de base amplia, muy diferente a las características poblacionales nacionales ${ }^{(6)}$. En un estudio realizado en el 2006 con una muestra representativa de 478 mujeres en edad fértil y 465 niños aguarunas, se encontró que la prevalencia de desnutrición crónica en niños fue de 33,4\%, encontrándose asociación estadística entre el distrito de residencia y la desnutrición crónica en niños ${ }^{(7)}$.

La prevalencia de desnutrición crónica infantil es heterogénea en el Perú. Según la Encuesta Demográfica y de Salud Familiar (ENDES) 2013 del INEI, la desnutrición crónica en menores de cinco años a nivel nacional es de $17,5 \%$ (de acuerdo al patrón de referencia de la OMS). Según la zona geográfica se evidencia que la desnutrición crónica en la selva es de $24,1 \%$, mientras que en Lima Metropolitana es de 4,1\%. Los departamentos con mayor prevalencia de desnutrición crónica son Huancavelica $(42,4 \%)$, Cajamarca $(35,6 \%)$, Loreto (27,7\%), Apurímac (29\%), Ayacucho (29\%), Amazonas (27,1\%) y Ucayali $(24,5 \%)^{(8)}$.

El tema indígena tiene que ser visto no solo como un problema de los indígenas, sino del Estado peruano. Un desarrollo desde la perspectiva indígena puede ser posible con la participación directa de los propios pueblos indígenas. Algunas de las exclusiones históricas a los indígenas son las brechas en lo referente al acceso a los servicios públicos: salud, vivienda, electrificación, las limitaciones de cobertura y calidad del sistema educativo, los niveles bajos de bienestar socioeconómico, problemas como la discriminación étnico-cultural, la inoperancia del sistema judicial, la desigualdad de oportunidades, entre otras, que no tienen ni gozan como los demás sectores sociales del Perú. También hay que señalar que, el Estado en vez de incorporar los temas indígenas dentro del Plan de Gobierno, los excluye ${ }^{(9-12)}$.

El objetivo del presente estudio fue determinar la prevalencia de desnutrición crónica y anemia en niños menores de 5 años de hogares indígenas de la región amazónica del Perú, en el año 2013.

\section{MÉTODOS}

La presente investigación corresponde a un estudio secundario de los datos de la Encuesta Demográfica y de Salud Familiar (ENDES) $2013^{(13)}$, del Instituto Nacional de Estadística e Informática (INEI), la cual se realizó de marzo a diciembre de 2013. La ENDES es una encuesta transversal, probabilística, de áreas, estratificada, bietápica e independiente en cada departamento. La representatividad de la muestra es a nivel nacional; además, el diseño estratificado permite obtener resultados representativos para las zonas de residencia urbana o rural y para cada uno de los 24 departamentos del país.
Para nuestro análisis se ha aplicado como criterio de inclusión las familias residentes en la región natural Selva, en las cuales el idioma es lengua nativa (quechua, aymara u otra lengua nativa, según codificación de la ENDES). Es a partir de este criterio que en la base de datos se encuentra a 334 niños menores de 5 años y aplicando los factores de ponderación correspondientes de la ENDES 2013, se tiene una frecuencia ponderada de 172 niños.

Los indicadores nutricionales evaluados fueron desnutrición crónica y anemia. Se consideró desnutrición crónica los valores $Z$ para el indicador talla para la edad < -2 DS según los valores de referencia de la OMS. Las mediciones antropométricas fueron realizadas por personal debidamente capacitado por el INEI, empleándose instrumentos estandarizados. Para la longitud (niños de 0 a 23 meses de edad) y estatura (niños a partir de 2 años) se emplearon tallímetros certificados por consultores de UNICEF. Se definió como anemia los valores de hemoglobina $<11 \mathrm{~g} / \mathrm{dL}$, ajustada por altitud. La determinación de hemoglobina se hizo mediante el sistema HemoCue ${ }^{\circledR}$.

Además, se caracterizó la situación socio-demográfica de la muestra según las siguientes variables: sexo del menor, edad del menor en meses, educación de la madre (ninguna, primaria, secundaria y superior), zona de residencia (urbana o rural), región (Amazonas, Loreto, Ucayali, Junín, Puno, Ayacucho, Cusco, Pasco, Madre de Dios y Huánuco), nivel económico, conexión domiciliaria de agua potable, conexión domiciliaria de desagüe, material predominante del piso de vivienda (tierra, madera, cemento y otros), diarrea durante las dos últimas semanas, tos durante las dos últimas semanas y tratamiento antiparasitario los últimos seis meses.

El análisis fue realizado tomando en cuenta las recomendaciones de ponderación para las ENDES. El análisis estadístico se realizó con el programa SPSS versión 21,0. Se elaboró tablas de distribuciones de frecuencia (abso- 
lutas y porcentuales) para las variables categóricas y se elaboraron histogramas para representar la distribución de los valores de los puntajes $Z$ del indicador talla/edad y niveles de hemoglobina ajustados por altitud. Para analizar el nivel de asociación entre la desnutrición crónica y anemia en relación al sexo y edad del niño se empleó la prueba de independencia chi cuadrado, con un nivel de significación de 5\%.

El método de recolección de datos de la ENDES fue mediante entrevista directa, con personal debidamente capacitado y entrenado para tal fin, aplicando el correspondiente consentimiento informado y tomando en cuenta las demás consideraciones éticas de investigación.

\section{RESULTADOS}

Los menores de cinco años incluidos en el presente análisis fueron mayoritariamente mujeres $(56,5 \%)$, con madres que tenían un nivel de educación primaria $(60,5 \%)$, residentes de zonas rurales $(94,2 \%)$ y procedentes de Amazonas $(29,0 \%)$, Loreto $(27,2 \%)$ y Ucayali $(23,9 \%)$; la distribución por edad tuvo un comportamiento homogéneo (tabla $1)$.

En relación al nivel socio-económico y características de la vivienda (tabla 2), se observa que el 94,5\% correspondió al quintil más pobre, según la clasificación del nivel de pobreza que realiza el INEI según quintiles; cabe destacar que ninguno de los hogares se ubicó en el quintil rico o muy rico. En cuanto a la conexión domiciliaria de agua potable, solo $25,4 \%$ contaba con este servicio, y sobre la conexión domiciliaria de desagüe, únicamente 3,9\% tenía el servicio. Respecto al material de piso de la vivienda, el 68,3\% era de tierra, mientras que solo en $4,6 \%$ era de cemento.

$\mathrm{Al}$ analizar la presencia de desnutrición crónica y anemia en niños menores de cinco años, se estimó que la prevalencia de desnutrición crónica era de $43,0 \%$ (IC95\% 35,5\% a 50,5\%)
Tabla1. Características generales de la muestra de estudio, niños menores de 5 años de hogares indigenas de la región selva, Perú 2013.

\begin{tabular}{|c|c|c|c|}
\hline Característica & & Frecuencia & Porcentaje \\
\hline \multirow{2}{*}{ Sexo } & Masculino & 75 & 43,5 \\
\hline & Femenino & 97 & 56,5 \\
\hline \multirow{5}{*}{ Edad (meses) } & Menor de 12 & 33 & 19,1 \\
\hline & 12 a 23 & 39 & 22,5 \\
\hline & 24 a 35 & 35 & 20,3 \\
\hline & 36 a 47 & 31 & 17,9 \\
\hline & 48 a 59 & 35 & 20,2 \\
\hline \multirow{4}{*}{$\begin{array}{l}\text { Educación } \\
\text { de la madre }\end{array}$} & Ninguna & 23 & 14,0 \\
\hline & Primaria & 99 & 60,5 \\
\hline & Secundaria & 40 & 24,8 \\
\hline & Superior & 1 & 0,7 \\
\hline \multirow{2}{*}{ Residencia } & Urbana & 10 & 5,8 \\
\hline & Rural & 162 & 94,2 \\
\hline \multirow{10}{*}{ Región } & Amazonas & 50 & 29,0 \\
\hline & Loreto & 47 & 27,2 \\
\hline & Ucayali & 41 & 23,9 \\
\hline & Junín & 14 & 7,9 \\
\hline & Puno & 8 & 4,5 \\
\hline & Ayacucho & 5 & 2,9 \\
\hline & Cusco & 4 & 2,1 \\
\hline & Pasco & 2 & 1,2 \\
\hline & Madre de Dios & 1 & 0,8 \\
\hline & Huánuco & 1 & 0,6 \\
\hline
\end{tabular}

y de anemia $43,5 \%$ (IC95\% 35,5\% a $51,5 \%)$. La presencia de diarrea en las últimas dos semanas afectó a 12,8\% de niños, el antecedente de tos en las últimas dos semanas se presentó en 32,2\% y recibieron algún medicamento antiparasitario en los últimos seis meses el $29,7 \%$ de la muestra (tabla 3).

La distribución de los puntajes $Z$ de la talla para la edad de los niños (figura
Tabla 2. Nivel económico y características de las viviendas de la muestra de estudio.

\begin{tabular}{|c|c|c|c|}
\hline \multicolumn{2}{|c|}{ Característica } & \multicolumn{3}{|c|}{ Frecuencia } & Porcentaje \\
\hline \multirow{2}{*}{ Nivel económico } & Más pobre & 162 & 94,5 \\
& Pobre & 8 & 4,9 \\
& Medio & 1 & 0,6 \\
\hline Conexión domiciliaria de agua & Sí & 44 & 25,4 \\
potable & No & 128 & 74,6 \\
\hline Conexión domiciliaria & Sí & 7 & 3,9 \\
desagüe & No & 165 & 96,1 \\
\hline \multirow{2}{*}{ Material del piso de la vivienda } & Tierra & 117 & 68,3 \\
& Madera & 27 & 15,9 \\
& Cemento & 8 & 4,6 \\
& Otros & 19 & 11,2 \\
\hline
\end{tabular}


Tabla 3. Indicadores nutricionales y de salud.

\begin{tabular}{|c|c|c|c|}
\hline \multicolumn{2}{|c|}{ Problema de salud } & Frecuencia & Porcentaje \\
\hline \multirow{2}{*}{ Desnutrición crónica } & No & 74 & 43,0 \\
& Sí & 66 & 57,0 \\
\hline \multirow{2}{*}{ Anemia } & Severa & 1 & 43,5 \\
& Moderada & 22 & 0,6 \\
& Leve & 42 & 14,9 \\
& Sin anemia & 85 & 28,0 \\
Diarrea las últimas dos semanas & Sí & 20 & 56,5 \\
\hline \multirow{2}{*}{ Tos las últimas dos semanas } & No & 138 & 12,8 \\
& Sí & 51 & 32,2 \\
\hline Recibió antiparasitarios los últimos & No & 108 & 67,8 \\
\hline 6 meses & Sí & 47 & 29,7 \\
& No & 111 & 70,3 \\
\hline
\end{tabular}

1) presentó una distribución aproximadamente simétrica, con una media de $-1,82$ y mediana de $-1,80$ puntos $\mathrm{Z}$. El histograma correspondió a una población predominantemente deficitaria, pues la ubicación de la campana en el eje de las abscisas es proporcionalmente mayor hacia la izquierda del valor cero. $\mathrm{Si}$ consideramos el número de niños que presentaron puntajes $Z$ entre -2 y -1 , el porcentaje de niños en riesgo de

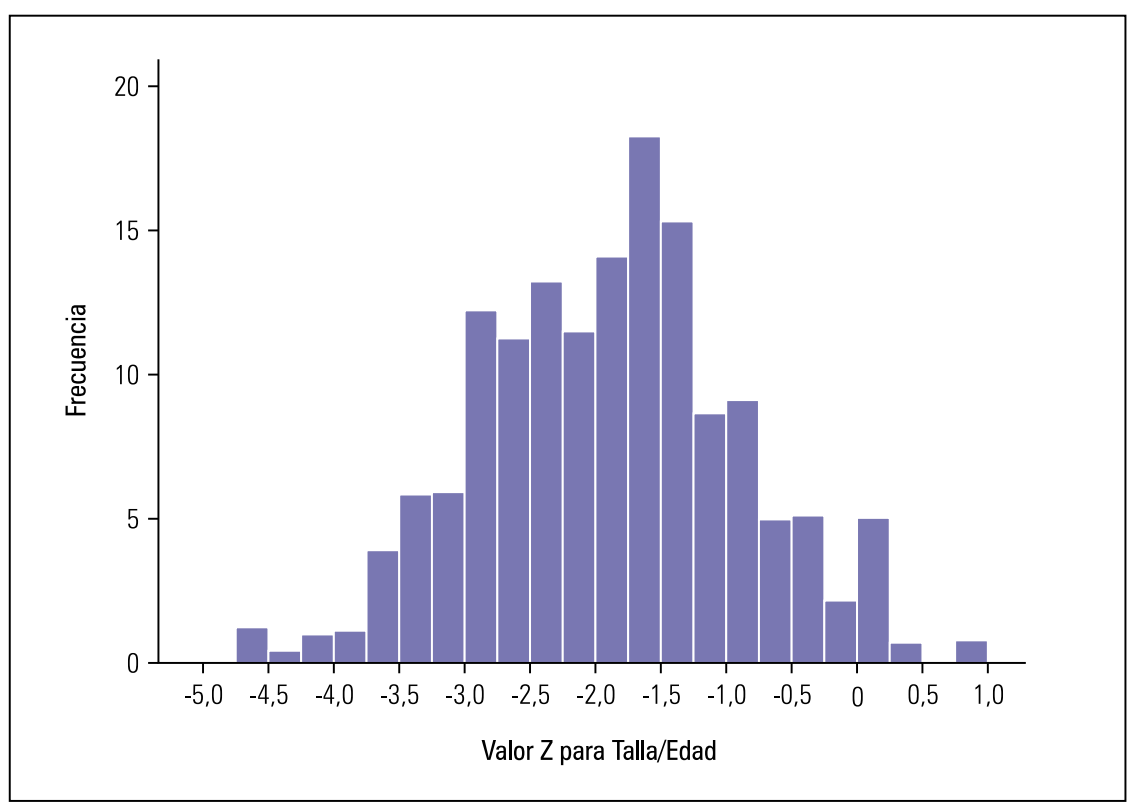

Figura 1. Distribución de los valores $Z$ de la talla para la edad de la muestra de estudio. desnutrición fue elevado, pues en este rango se encuentra alrededor de $30,2 \%$ de los niños. Además, un importante grupo de niños evidenció desnutrición crónica severa $(12,4 \%)$, al tener valores $\mathrm{Z}<-3,0$. En cuanto al nivel de hemoglobina (figura 2), se aprecia una distribución asimétrica de cola inferior, con una media de $10,9 \mathrm{~g} / \mathrm{dL}$ y una mediana de $11,1 \mathrm{~g} / \mathrm{dL}$; el valor mínimo registrado fue $5,5 \mathrm{~g} / \mathrm{dL}$.
$\mathrm{Al}$ analizar la frecuencia de desnutrición crónica y anemia según sexo, se ha encontrado una mayor prevalencia en el sexo masculino $(48,6 \%$ y $45,6 \%$, respectivamente) frente al sexo femenino ( $39,2 \%$ y $41 \%$, respectivamente), pero estas diferencias no son estadísticamente significativas (tabla 4). Respecto al análisis por grupos de edad, la desnutrición crónica fue mayor en los niños de 12 a 23 meses, con 53,8\%, pero no se estableció una asociación estadísticamente significativa. En cuanto a la anemia y edad, se aprecia que la totalidad de niños menores de 12 meses presentaron anemia, disminuyendo la prevalencia conforme avanzaba la edad del niño, siendo esta tendencia significativa (tabla 4).

\section{DISCUSIÓN}

En la población de niños peruanos menores de 5 años, la prevalencia de DC fue $17,5 \%$, con $37,6 \%$ en el quintil de riqueza inferior (más pobres) y 39,8\% en la zona rural. En nuestro análisis, la prevalencia de DC fue $43,0 \%$, siendo notoria y distante la diferencia.

Un estudio realizado en el 2004 en la comunidad de los Aguarunas ${ }^{(7)}$, se encontró que la prevalencia de desnutrición crónica fue de $33,4 \%$, hallándose asociación estadística entre el distrito de residencia y la desnutrición crónica en niños. En nuestro análisis se halló que más de $90 \%$ de la población indígena residía en la zona rural y en su mayoría no contaba con los servicios básicos de agua y desagüe, lo que no favorecía condiciones mínimas de salubridad y de calidad de vida, lo que también es destacado por el Banco Mundial ${ }^{(14)}$, que manifiesta que las disparidades a lo largo del país siguen siendo altas, particularmente entre las áreas rural y urbanas. De igual manera encontramos que más del $90 \%$ perteneció al nivel económico más pobre, lo que limitaría el acceso a una alimentación balanceada y variada, y a la vez también es considerada como factor altamente determinante en la prevalencia de DC según diferentes estudios ${ }^{(9,15)}$. 


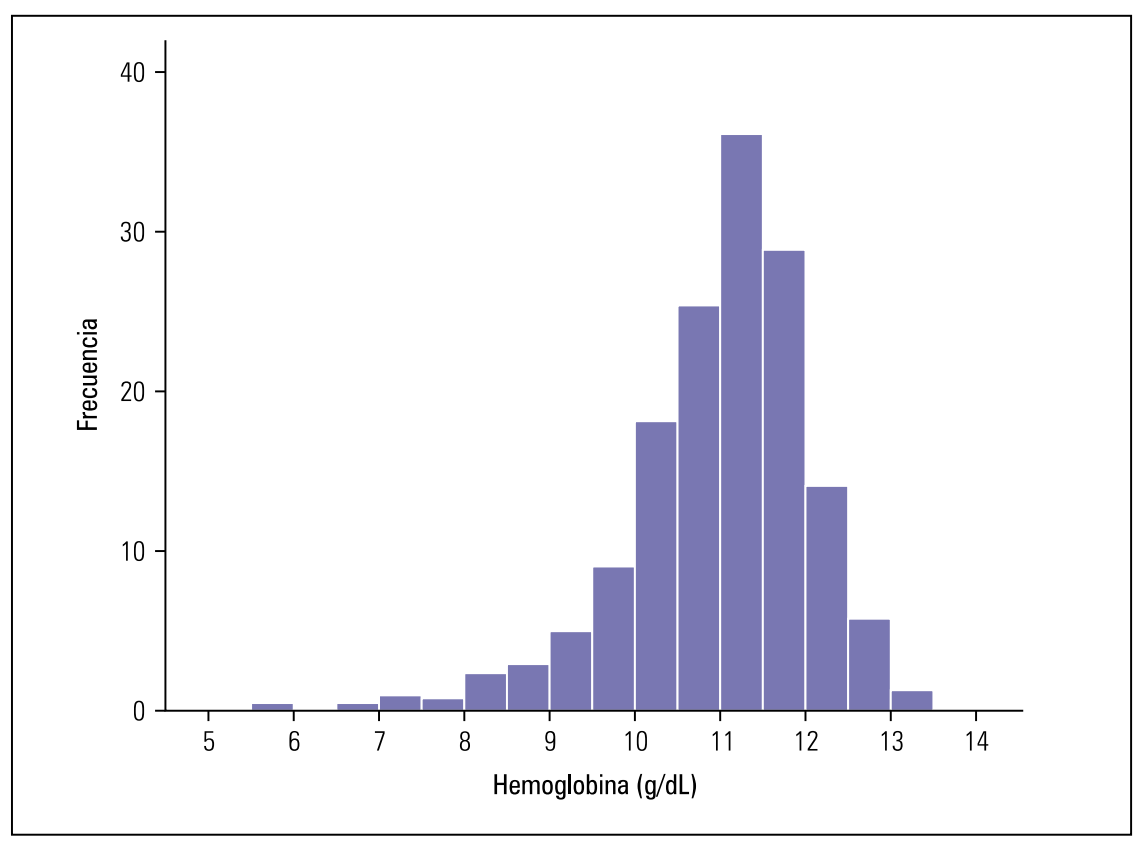

Figura 2. Distribución del nivel de hemoglobina $(\mathrm{g} / \mathrm{dL})$.

En un estudio de Salud y Nutrición de niños menores de 5 años de Bagua y Condorcanqui, Amazonas ${ }^{(17)}$, se encontró un 56,2\% de niños con desnutrición crónica y $51,3 \%$ con anemia, siendo la DC infantil más del doble en los niños indígenas respecto de los no indígenas, mientras que más de $70 \%$ de los niños indígenas menores de 2 años eran anémicos. Estos hallazgos incluso son superiores a los encontrados en nuestro análisis y refuerzan una realidad que se presumía, que la población infantil indígena presenta uno de los más altos niveles de desnutrición crónica, pobreza y vulnerabilidad de todo el país. Lo que en consecuencia afecta

la productividad de su población y sus capacidades de desarrollo socioeconómico.

La alta prevalencia de anemia en nuestro estudio superó en 13,5 puntos porcentuales a lo hallado a nivel nacional (34\%), siendo preocupante encontrar al total de niños menores de 12 meses con anemia; en otros estudios no se halla valores similares, lo cual es preocupante para nuestra población de menores de 12 meses. En un trabajo realizado por Echague y col ${ }^{(18)}$ en menores de 5 años de comunidades indígenas en Paraguay, encontraron $74,2 \%$ de anemia, observándose mayor prevalencia en el sexo masculino que en

Tabla 4. Desnutrición crónica y anemia según sexo y edad.

\begin{tabular}{|c|c|c|c|c|c|}
\hline \multicolumn{2}{|c}{ Característica } & $\begin{array}{c}\text { Desnutrición } \\
\text { crónica }\end{array}$ & Valor * $^{*}$ & Anemia & Valor $p^{*}$ \\
\hline \multirow{3}{*}{ Sexo } & Masculino & 48,6 & 0,215 & 45,6 & 0,568 \\
& Femenino & 39,2 & & 41,0 & \\
\hline \multirow{5}{*}{ Edad } & Menor de 12 & 27,3 & 0,257 & 100,0 & $<0,001$ \\
(meses) & 12 a 23 & 53,8 & & 50,0 & \\
& 24 a 35 & 45,7 & & 29,4 & \\
& 36 a 47 & 43,3 & & 35,5 & \\
& 48 a 59 & 44,1 & & 32,4 & \\
\hline
\end{tabular}

* Prueba chi cuadrado. el femenino, situación que también se halló en nuestro análisis. Estos valores altos de anemia en nuestro análisis se explicarían por el consumo deficitario de alimentos fuentes de hierro, lo cual fue hallado por Huamán y Valladares ${ }^{(7)}$ en un estudio realizado en una población de Aguarunas en donde se consume diariamente yuca y plátanos, y con alguna frecuencia semanal arroz, carachama, gusano, huevo de gallina y verduras como la chonta y sachaculantro. La presencia de desnutrición crónica crea las condiciones necesarias para la anemia, perjudicando en mayor manera al organismo. Por tanto, si un niño tiene una desnutrición por largo tiempo dificultará la producción de eritropoyetina y por consiguiente la producción de glóbulos rojos, provocando de esta manera anemia; aunado a esto, el bajo consumo de alimentos ricos en hierro, enteroparasitosis, entre otras son determinantes de la salud que también influyen en gran medida.

La presencia de parásitos también explicaría esta alta prevalencia de anemia, teniendo en cuenta que en nuestro análisis menos de la tercera parte de los niños recibió la profilaxis antiparasitaria en los últimos seis meses anterior al estudio, y habiéndose encontrado en el estudio en Bagua en el $2012^{(17)}$ que $64,2 \%$ de los niños evaluados presentó enteroparásitos patógenos.

En un trabajo que analiza las tendencias en materia de desnutrición y anemia en menores de 5 años peruanos y su asociación con algunos factores determinantes en el período 2000-2011, se encontró que los factores determinantes tienen incidencia en la prevalencia de DC. En el presente estudio se reveló diferencias estadísticamente significativas en la educación de la madre, vivir en la Sierra o en mayor altitud, tener dos o más hijos en el hogar, la posición en el orden de nacimientos (a partir del tercer hijo) y haber presentado diarrea en los 15 días previos a la encuesta ${ }^{(13)}$.

Nuestro trabajo presenta como una de sus mayores limitaciones que al usar datos de la ENDES provee información 
confiable solo para grandes dominios geográficos, por lo que puede alimentar con información a nivel nacional para el gobierno central, pero ya con menor confiabilidad para los gobiernos regionales y ningún dato para los gobiernos locales. Por esta razón es necesario potenciar los sistemas de registro para un conjunto de variables claves que permita proveer información por lo menos hasta el nivel de provincias y distritos, sin dejar de lado a la población indígena. A esto se suma que los diversos grupos étnicos en nuestro país presentan variaciones nutricionales en función a la diversidad en la producción local de alimentos y prácticas culturales, por lo que debería desarrollarse más investigaciones específicas.

En conclusión, la desnutrición crónica infantil es elevada en niños de hogares de familias indígenas, a pesar de que a nivel nacional la prevalencia ha disminuido en los últimos años. Las intervenciones que se han realizado hasta el momento no han logrado disminuir la prevalencia de DC. De igual manera encontramos una alta prevalencia de anemia infantil en comunidades indígenas. Se observa el abandono de parte del Estado, siendo evidentes las grandes desigualdades en la situación de pobreza, servicios básicos y salud de los niños indígenas. La inversión para erradicar la desnutrición es un imperativo ético, de justicia social y de respeto a los derechos humanos; pero, es también una forma sostenible de invertir para la su- peración de la pobreza, por aumentar el crecimiento de la economía nacional.

Es necesaria una intervención que contribuya a fortalecer los sistemas de salud que atienden a los niños que viven en situación de inseguridad y exclusión en la población indígena y contribuir de esta manera al cierre de brechas en salud que persisten en el país.

\section{REFERENCIAS BIBLIOGRÁFICAS}

1. UNICEF, Ministerio de Salud, USAID Perú. Un buen inicio de la vida. Perú, 2006; 76 pp.

2. Beltrán A, SeinfeldJ. Identificando estrategias efectivas para combatir la desnutrición infantil en el Perú. Apuntes Centro de Investigación de la Universidad del Pacifico. 2011.

3. Alcázar L, Ocampo D, Huamán-Espino L, Aparco JP. Impacto económico de la desnutrición crónica, aguda y global en el Perú. Rev Peru Med Exp Salud Publica. 2013;30(4):569-74.

4. Mayor P, Bodmer R, Pueblos Indígenas de la Amazonía Peruana, Centro de Estudios Teológicos de la Amazonía (CETA). Iquitos, Perú. Julio 2009.

5. Contreras H, Pérez $P$, Huapaya $O$, Chacón $H$, Champin D, Adrianzén L, Arévalo C, Torres S, Black C. La salud en las comunidades nativas amazónicas del Perú. Rev Perú Epidemiol. 2014;18(1).

6. Perú, Instituto nacional de Estadística e Informática. II Censo de Comunidades Indigenas de la Amazonia Peruana 2007. Lima: INEI; 2007.

7. Huamán L, Valladares C. Estado nutricional y características del consumo alimentario de la población Aguaruna. Amazonas, Perú 2004. Rev Perú Med Exp Salud Publica. 2006;23(1):12-21.

8. Perú, Instituto Nacional de Estadistica e Informática Perú (INEI). Ficha técnica: Encuesta demográfica y de salud familiar- ENDES 2012. Lima: INEl;2012

9. Arpasi P. Comunidades campesinas y comunidades nativas. Fondo Editorial del Congreso del Perú. Lima 2005:186 pp.

10. Banco Interamericano de Desarrollo: Sobre exclusión social. Consulta: 08 de Julio del 2011. Disponible en: http://www.iadb.org/sds/soc/ site_3094_s.htm.
11. GRADE. Etnicidad, pobreza y exclusión social: la situación de la población indigena en Perú. Reporte de investigación elaborado por Néstor Valdivia para el Banco Mundial, Lima. 2002.

12. Sobrino M, Gutiérrez C, Cunha AJ, Dávila M, Alarcón J. Desnutrición infantil en menores de cinco años en Perú: tendencias y factores determinantes. Rev Panam Salud Pública. 2014;35(2):104-12.

13. Perú, Instituto Nacional de Estadística e Informática Perú (INEI). Ficha técnica: Encuesta demográfica y de salud familiar- ENDES 2013. Lima: INEI; 2013.

14. Banco Mundial. Informe sobre Perú Panorama General, Abril 10, 2014. Disponible en: http://www. bancomundial.org/es/country/peru/overview.

15. Ibáñez N, Jara C, Guerra A, Díaz E. Prevalencia del enteroparasitismo en escolares de comunidades nativas del Alto Marañón, Amazonas, Perú. Rev Perú med exp salud publica. 2004 Jul;21(3):126-33.

16. Martínez N. Hambre y desigualdad en los Países Andinos: la desnutrición y la vulnerabilidad alimentaria en Bolivia, Colombia, Ecuador y Perú. CEPAL Naciones Unidas: Serie politicas sociales. Santiago, 2005 Dic: 95 pp.

17. Diaz A, Arana A. Informe Técnico: Estado de salud y nutrición de los niños menores de 5 años pertenecientes a las poblaciones indigenas y no-indigenas de Bagua y Condorcanqui en la Región Amazonas 2012. OPS/OMS Perú 2014 Jul.

18. Echague G, Sosa L, Diaz V y col. Anemia en niños indígenas y no indígenas menores de 5 años de comunidades rurales del Departamento de Caazapá. Pediatr (Asunción). Abr 2013;40(1):19-28.

Artículo recibido el 15 de octubre de 2014 y aceptado para publicación el 8 de noviembre de 2014.

Conflictos de intereses: Los autores expresan no tener algún conflicto de interés.

Autor corresponsal:

Janet Flores Bendezú

Programa de Segunda Especialidad en Nutrición

Pública, Facultad de Medicina, UNMSM

Celular: 99873-1491

Correo electrónico: janflor@peru.com 